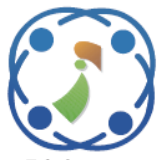

\title{
Intelligent System for Parasitized Malaria Infection Detection Using Local Descriptors
}

\author{
Yossra H. Ali $^{1} \quad$ Suhaila N. Mohammed ${ }^{2 *}$ \\ ${ }^{1}$ Computer Sciences Department, University of Technology, Baghdad, Iraq \\ ${ }^{2}$ Department of Computer Science, College of Science, University of Baghdad, Baghdad, Iraq \\ * Corresponding author’s Email: suhailan.mo@sc.uobaghdad.edu.iq
}

\begin{abstract}
Malaria is a curative disease, with therapeutics available for patients, such as drugs that can prevent future malaria infections in countries vulnerable to malaria. Though, there is no effective malaria vaccine until now, although it is an interesting research area in medicine. Local descriptors of blood smear image are exploited in this paper to solve parasitized malaria infection detection problem. Swarm intelligence is used to separate the red blood cells from the background of the blood slide image in adaptive manner. After that, the effective corner points are detected and localized using Harris corner detection method. Two types of local descriptors are generated from the local regions of the effective corners which are Gabor based features and color based features. The extracted features are finally fed to Deep Belief Network (DBN) for classification purpose. Different tests were performed and different combinations of feature types are attempted. The achieved results showed that when using combined vectors of local descriptors, the system gives the desired accuracy which is $100 \%$. The achieved result demonstrates the effectiveness of using local descriptors in solving malaria infection detection problem.
\end{abstract}

Keywords: Malaria infection detection, Particle swarm intelligence, Corner detection, Deep belief network (DBN), Gabor filter.

\section{Introduction}

Malaria is a life-threatening mosquito-borne disease produced by the Plasmodium parasite that attacks Red Blood Cells (RBCs). An estimated 3.2 billion people worldwide are at high risk (> 1 in 1000 chances of malaria in a year) [1]. In 2019, an estimated 228 million cases and 405,000 fatalities worldwide were reported by the World Health Organization (WHO) report [2].

Every year, hundreds of millions of blood films are tested for malaria, which includes a specialized microscopist manually counting parasites and infected RBCs. Though, microscopic detection is not comprehensive and relies heavily on the microscopist's expertise and skills. It is common for microscopists to operate in isolation in low-resource environments, without a rigorous system that can guarantee the preservation of their skills and indeed the reliability of diagnosis. This will lead to erroneous diagnostic decisions in this field [3].

Computer vision methods for the diagnosis and detection of malaria constitute a relatively new field for early detection of malaria where the medical imaging has the ability in overcoming the problems associated with manual analysis carried out by human visual inspection of blood smears [4].

The entire malaria infection detection process includes the ability to use visual information to distinguish between non-parasitic red blood cells, and the malarial parasites [5].

In general, a framework for automated cell microscopy comprises a set of main processing stages that can act as a guideline. Usually, the first stage is the acquisition of digital blood smear images, which primarily depends on the devices and materials being used. After that, most systems perform one or more pre-processing methods to eliminate noise and to normalize lighting and color variations inherent in the 
process of acquiring images. The next stage typically involves the identification and segmentation (outlining) of individual blood cells and perhaps other items, such as parasites or platelets that can be visible in a blood slide image. After that, the discriminated features are generated and extracted to work as a final descriptor of blood slide image. In the last stage, a decision is made about the existence of malaria infection based on the computed features using some classifiers [1].

Different works have been conducted to build a system for malaria infection detection task. Mushabe et al. (2013) [6] used morphological operations and histogram-based thresholding to isolate the RBCs. The RBCs are classified using a Bayesian classifier with the values of pixel color values as attributes. The achieved sensitivity was $98.5 \%$ and specificity was 97.2\%. Savkare and Narote (2015) [7] used Median filter for smoothening blood slide image and Laplacian filter for sharpening. After that, Otsu's threshold is used for RBCs segmentation. Watershed transform is used for separating overlapped cells. Color, textual and shape based features are generated from the RBCs for infected and non-infected cells classification. Correct detection rate, sensitivity and specificity were $98.66 \%, 98.94 \%$, and $96.12 \%$, respectively. Bashir et al. (2017) [8] extracted set of features based on color intensity and fed them into Artificial Neural Network (ANN) for classification purpose. The accuracy of $99.68 \%$ was achieved. Pan et al. (2018) [9] used Convolutional Neural Network (CNN) for malaria detection and obtained accuracy equals to 95\%. Quan et al. (2020) [10] proposed a classification network named Attentive Dense Circular Net (ADCN) which based on CNN. ADCN gave accuracy (97.47\%), sensitivity (97.86\%) and specificity $(97.07 \%)$.

Despite of different works have been conducted in identification the infected blood cells, there is still no effective descriptor set that can distinguish the positive malaria infected blood smears from the negative ones. The main motivation of the paper is the fact that the corner-based local descriptors have not been reported in malaria infection detection problem. To fill this research gap, this paper aims at finding the application of Gabor and color based features within the local regions of points of interest during blood slide image analysis. The objective of the paper is to design an intelligent system for malaria infection detection as a supplementary tool for microscopists. The main contributions of this work can be summarized with the following points:

1. Swarm intelligence is employed for purpose of slide image segmentation in adaptive manner.
2. Corner based descriptors are proposed to measure the efficiency of local features type in malaria detection problem.

3- A comparison is made between Gabor based features and color based features when used to describe the local regions.

The remaining of the paper is organized as follows: Section 2 shows the detailed description of the proposed system for malaria infection detection. Section 3 illustrates the experimental results that are achieved when applying the proposed system on real blood slide images. Finally, work conclusions and ideas for future work are provided in section 4 .

\section{The proposed method}

The proposed intelligent system for Malaria infection detection involves five main stages: preprocessing of blood slide image, PSO-based automatic segmentation, effective corners localization, local feature extraction and Malaria infection detection. The block diagram of the proposed system is shown in Fig 1.

\subsection{Preprocessing of blood slide image}

Preprocessing of blood slide image is an initial stage that is required for improving image color quality and image brightness adjustment. After preprocessing stage, the resulted image is more suitable for further stages of the proposed system. This stage consists of two steps which are contrast enhancement and bright intensity removal.

\subsubsection{Contrast enhancement}

In contrast enhancement step, the intensities of the blood slide image are redistributed to cover the range of possible intensities of grey scale. The slide image is firstly converted into grey scale version before contrast enhancement process. Given the image pixel $\operatorname{Img}(x, y)$, the minimum $\operatorname{Img}_{\text {Min }}$ and

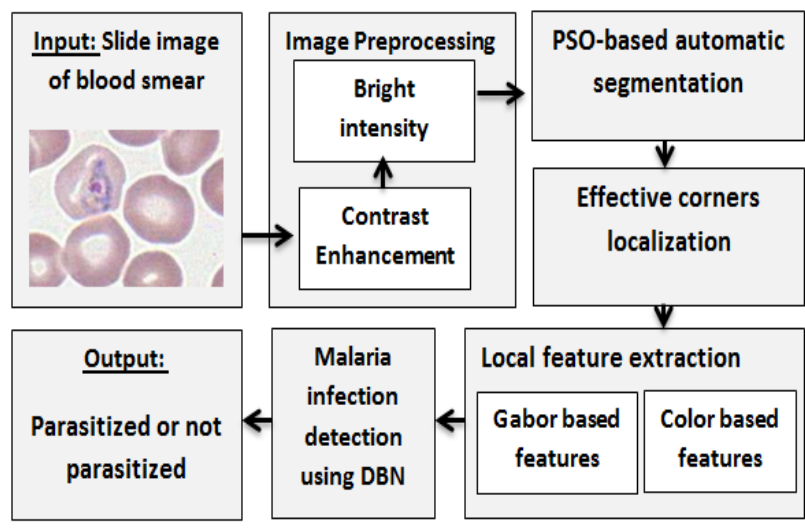

Figure. 1 The block diagram of the proposed system 
maximum $I m g_{\text {Max }}$ intensity values in $I m g$, the enhanced pixel $\operatorname{Img}_{\text {con }}(x, y)$ can be calculated using the following equation [11]:

$$
\operatorname{Img}_{\text {con }}(\mathrm{x}, \mathrm{y})=255 \times\left(\frac{\operatorname{Img}(\mathrm{x}, \mathrm{y})-\operatorname{Img}_{\text {Min }}}{\operatorname{Img}_{\text {Max }}-\operatorname{Img}_{\text {Min }}}\right)
$$

\subsubsection{Bright intensity removal}

To reduce the effect of high intensity values during segmentation process, a bright threshold is used to drop out the pixels' intensities which are greater than or equal to bright threshold to become equal to zero.

\subsection{PSO-based automatic segmentation}

Particle Swarm Intelligence (PSO)-based automatic thresholding method that proposed by [12] has been used in this work to conduct image segmentation. This method aims at automated the segmentation process by finding an adaptive threshold value that is changed from one image to another according to grey scale distribution of the RBCs and the background in the slide image. To find the optimal threshold value, the behaviour of particle swarm during flying is exploited. The PSO aims at minimizing the following objective function:

$$
J\left(a, t^{*}\right)=\operatorname{Min}_{0 \leq t \leq L-1} J(\beta, t)
$$

Where $J(\beta, t)$ can be obtained using Li [13] method which represents the variance between the RBCs and the background of the image as following [9]:

$$
J(\beta, t)=\beta\left(\sigma_{1}^{2}(t)+\sigma_{2}^{2}(t)\right)+(1-\beta) \sigma_{D}^{2}(t)
$$

and

$$
\sigma_{D}^{2}(t)=\sigma_{1}(t) \sigma_{2}(t)
$$

Where, $\beta$ represents the degree of transparency between the foreground (RBCs) and background, $\sigma_{D}^{2}(t)$ refers to the measure of the variance degree between the RBCs and the background, $\sigma_{2}(t)$ and $\sigma_{1}(t)$ are their standard deviation, respectively. At the end of the optimization process, the global best particle $\left(G_{b e s t}\right)$ will be considered as the optimal threshold value for the blood slide image under segmentation.

However; the image resulted from segmentation process consists of dark colored RBCs and a background with brighter colors. So, as a postprocessing operation, intensity complement is performed by turning the image into negative image
Table 1. Results of PSO-based automatic segmentation

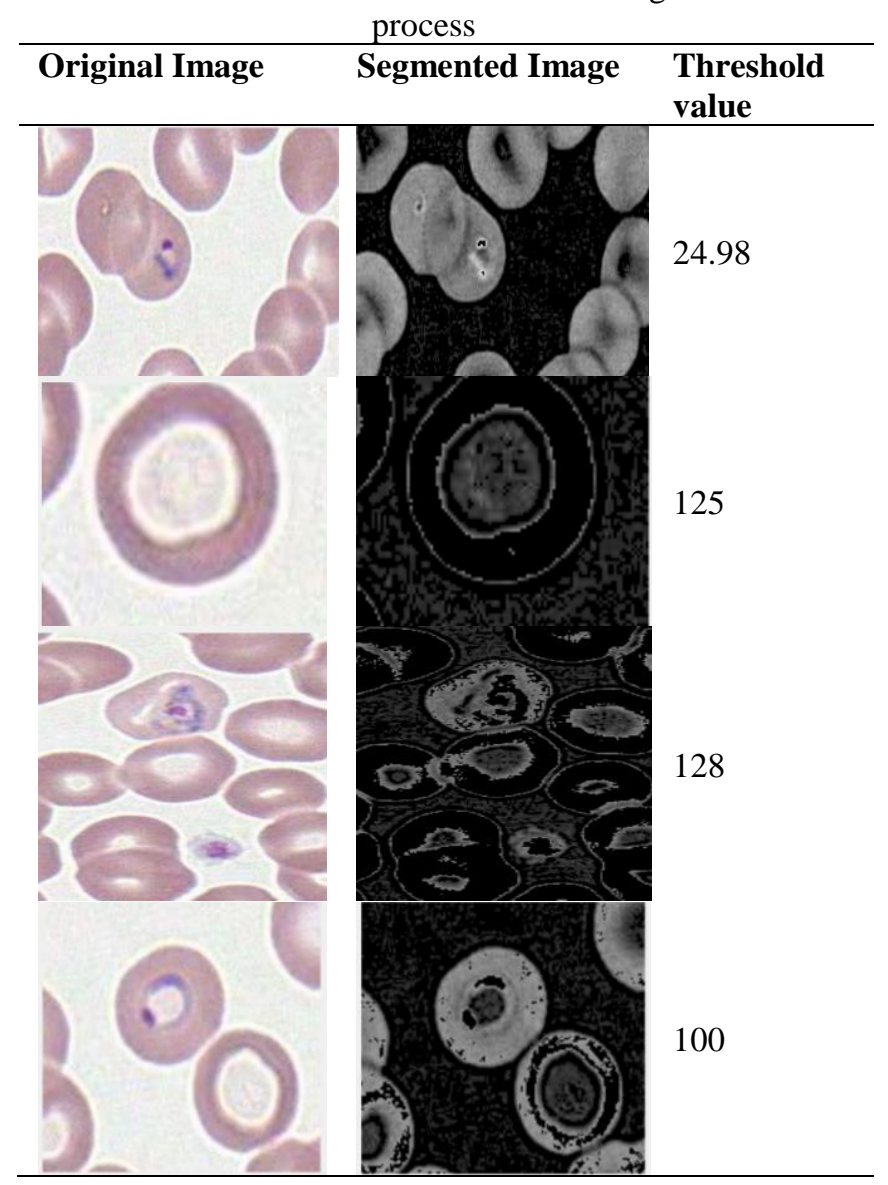

form. Once the complement process is done, the RBCs or parasite will turn brighter, and the background becomes darker. Table 1 shows results of segmentation process on four different blood slide images along with the adaptive threshold value that reached after optimization process of PSO.

\subsection{Effective corners localization}

Local based features have been proposed in this work to get insight into the deep local regions of the texture within the segmented slide image and retain the micro details that can lead to more strong descriptors. However; to generate such descriptors the effective local region must be localized first. The following steps are applied to find effective corner points in the slide image that is resulted from segmentation process:

(1) Corner detection: The method that is proposed by Chris Harris and Mike Stephens [14] is employed in this work to find corner's coordinates. Harris detects corner by taking into account the differential of the corner score with respect to direction. A shifting window is opened in any direction and the point that gives a large change in the intensity is registered as a corner. To find Harris corners, the products of 
derivatives at every pixel in the image (I) are firstly calculated using Eqs. (5) and (6).

$$
\begin{aligned}
& I_{x}^{2}=I_{x} * I_{x} \\
& I_{y}^{2}=I_{y} * I_{y}
\end{aligned}
$$

Where $\left(I_{x}\right.$ and $\left.I_{y}\right)$ are the derivatives of the segmented slide image which are computed using Sobel gradient masks $\left(G_{x}\right.$ and $\left.G_{y}\right)$ as in the following equations:

$$
\begin{aligned}
& I_{x}=G_{\mathrm{x}} * I \\
& I_{y}=G_{y} * I
\end{aligned}
$$

Where,

$$
\begin{gathered}
G_{x}=\left[\begin{array}{lll}
-1 & 0 & +1 \\
-2 & 0 & +2 \\
-1 & 0 & +1
\end{array}\right] \\
G_{y}=\left[\begin{array}{ccc}
-1 & -2 & -1 \\
0 & 0 & 0 \\
+1 & +2 & +1
\end{array}\right]
\end{gathered}
$$

*represents convolution operation.

After that, the response of the detector $(R)$ at each pixel $I(x, y)$ is computed using Eq. (11).

$$
R=\operatorname{det}(M)-k(\operatorname{trace}(M))^{2}
$$

Where,

$$
M=\sum_{x, y} I(x, y)\left[\begin{array}{cc}
I_{x}^{2} & I_{x} I_{y} \\
I_{x} I_{y} & I_{y}^{2}
\end{array}\right]
$$

and $\operatorname{det}(M)$ is the determinant of the matrix that can be founded using the following equation:

$$
\operatorname{det}(M)=I_{x}^{2} \cdot I_{y}^{2}-I_{x} I_{y} \cdot I_{x} I_{y}
$$

$\operatorname{trace}(M)$ is the sum of diagonal elements and can be computed as:

$$
\operatorname{trace}(M)=I_{x}^{2}+I_{y}^{2}
$$

$k$ is a tunable parameter, it is usually selected from the range [0.04-0.06]. Finally, threshold the value of $\mathrm{R}$ using threshold value $(T)$ as follows:

$$
\operatorname{Decision}(R)=\left\{\begin{array}{cl}
\text { Corner } & \text { if } R>T \\
\text { Non }- \text { corner } & \text { otherwise }
\end{array}\right.
$$

(2) Non maximal suppression: In non-maximal suppression, the number of corner points that are generated using Harris method will be reduced to $C_{\text {Best }}$ points only. The main reason behind nonmaximal suppression step is to reduce the size of the resulted descriptor which in effect speeds up feature extraction task and increases the efficiency of the resulted model. The $C_{B e s t}$ points are selected using the following steps:

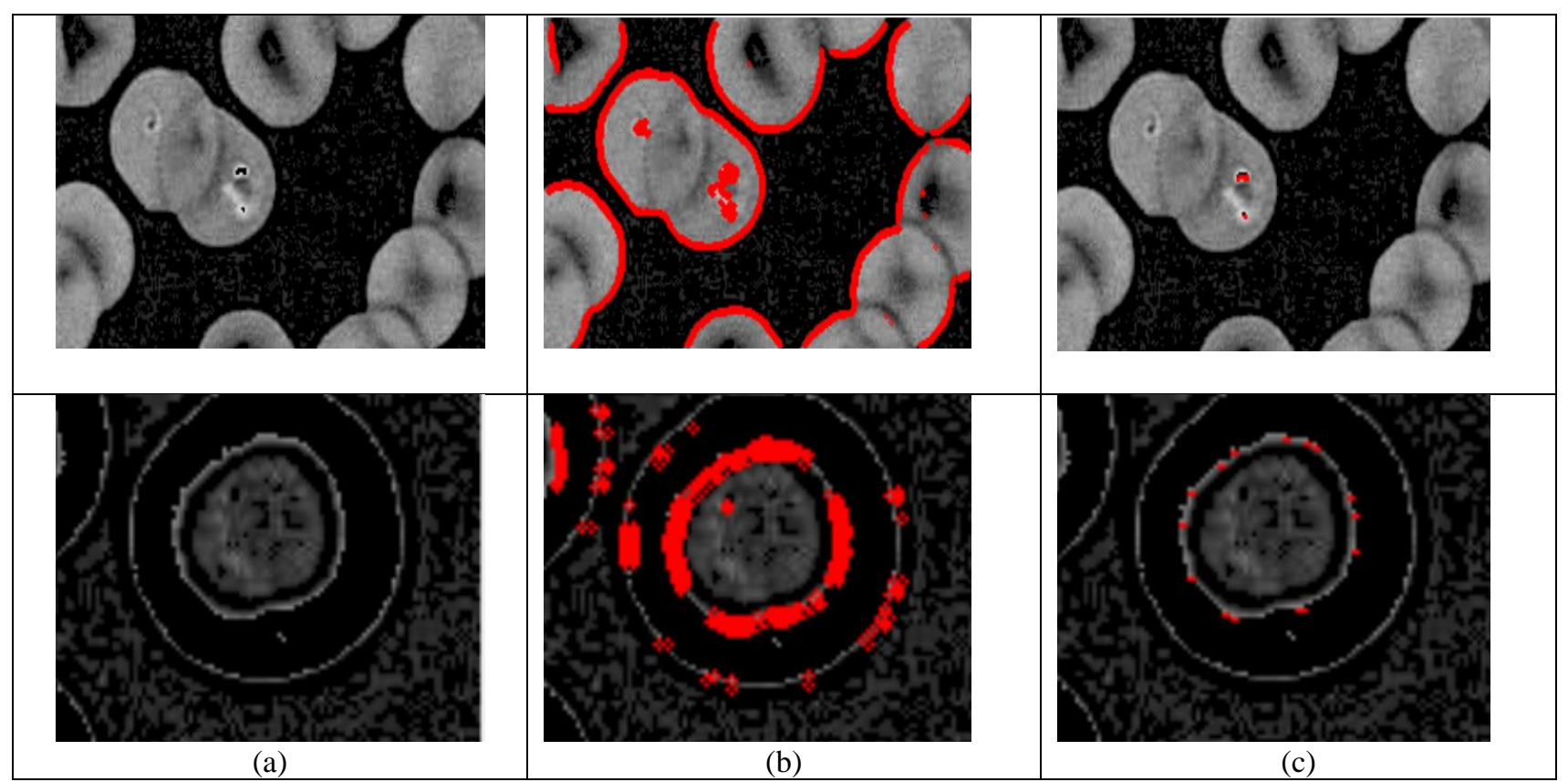

Figure. 2 Effective corner detection results with $C_{B e s t}=15$ : (a) slide image after segmentation, (b) corner detection result, and (c) non-maximal suppression result 
1. For each corner point $P(x, y)$ in the image $(I)$, the fitness value is computed by opening a window of size $\left(N_{w s} \times N_{w s}\right)$ around the point and applying the following equation:

$$
\begin{array}{r}
\text { Fitness }(P(x, y))=\sum_{m=0}^{N_{W s}} \sum_{n=0}^{N_{W s}} \mid I(x, y)- \\
I(x-m, y-n) \mid
\end{array}
$$

2. The corners are then sorted in ascending order based on their fitness value.

3. The first $C_{\text {Best }}$ points which give best fitness values are finally selected and considered as points of interest during feature extraction stage.

The results of applying Harris corner detection and non-maximal suppression with $C_{B e s t}=15$ are shown in Fig. 2 using two different blood slide images.

\subsection{Local feature extraction}

After localizing the effective corner points in blood slide image, two types of descriptors are generated: Gabor based features and color based features. However; these features are generated after opening a circular window with radius $R$ around each corner point and then the extracted features of all points are merged together to form the final feature vector for the given feature type. Fig. 3 shows examples of the opened circular windows around the finally selected effective points with different $R$ values.

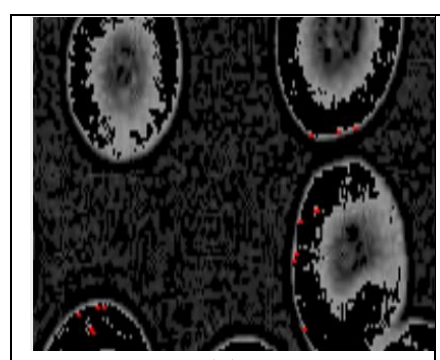

(a)

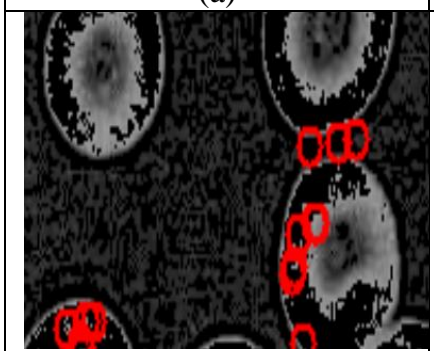

(c)

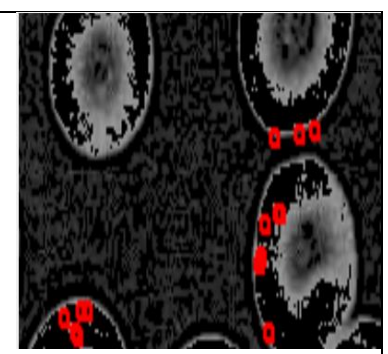

(b)

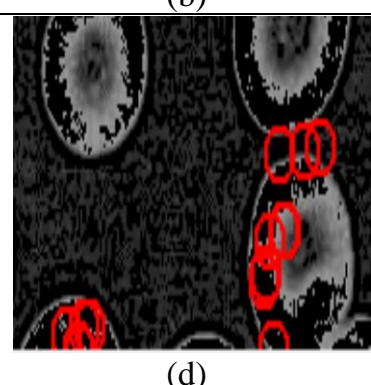

(d)
Figure. 3 Examples of the opened circular window around effective corner points: (a) effective corner points,

(b) opened circular windows with $R=5$, (c) opened circular windows with $R=10$, and (d) opened circular windows with $R=15$
A) Gabor based features

Gabor filters have gained substantial attention in image processing filed. A Gabor filter can be visualized as a sinusoidal plane of particular frequency and orientation, modulated by a Gaussian envelope. It can be written as [15]:

$$
G(x, y, \sigma, \theta)=\frac{1}{2 \pi \sigma^{2}} e^{-\frac{x^{2}+y^{2}}{2 \sigma^{2}}} e^{j 2 \pi(x \cos \theta+y \sin \theta)}
$$

where $\sigma$ and $\theta$ denote the bandwidth and orientation of Gabor filter, respectively, and $j=\sqrt{-1}$.

Since characteristics of certain RBCs in the slide image of blood smear can be approximated by Gabor filter, it is applied in the slide image to highlight these characteristics. After finding the Gabor magnitude of the slide image, a Run Length Matrix (RLM) is created for each opened circular window. The RLM is considered to be the basis from which the features are derived. RLM is a 2-D matrix used to describe the color intensity variance in the opened window, such that, each entry in the matrix contains the number of runs of a given length and intensity in that window. By dividing each entry in the matrix by the total number of RLM entries, a joint conditional probability density function $\mathrm{p}(\mathrm{i}, \mathrm{j})$ can be obtained from the matrix. The five characteristics below are generated from the RLM of each window [11]:

1. Short-Run Emphasis (SRE): If there are so many short runs in the window, this attribute will be high. SRE can be defined by the following equation:

$$
\mathrm{SRE}=\sum_{i=1}^{N \text { pixels }} \sum_{l}^{N \text { colors }} \frac{\mathrm{p}(\mathrm{i}, \mathrm{l})}{\mathrm{l}^{2}}
$$

2. Long-Run Emphasis (LRE): If there are too many long runs in the window, this feature will be high. LRE can be computed as follows:

$$
\mathrm{LRE}=\sum_{i=1}^{\text {Npixels }} \sum_{l}^{\text {Ncolors }} \mathrm{l}^{2} \mathrm{p}(\mathrm{i}, \mathrm{l})
$$

3. Gray-Level Non-uniformity $(G L N)$ : If there are too many number of runs with equal intensities in the window, this attribute will be high, which means the window having varying intensities. Eq. (20) is utilized to compute GLN.

$$
\mathrm{GLN}=\frac{\sum_{i=1}^{N \text { pixels }}\left(\sum_{l}^{\text {Ncolors }} \mathrm{p}(\mathrm{i}, \mathrm{l})\right)^{2}}{\sum_{i=1}^{N \text { pixels }} \sum_{l}^{\text {Ncolors }} \mathrm{p}(\mathrm{i}, \mathrm{l})}
$$

4. Run-Length Non-uniformity $(R L N)$ : This feature will be high when the window has repeated patterns. Eq. (21) is used to compute RLN. 


$$
\mathrm{RLN}=\frac{\sum_{l=1}^{\text {Npixels }}\left(\sum_{i}^{\text {Ncolors }} \mathrm{p}(\mathrm{i}, \mathrm{l})\right)^{2}}{\sum_{i=1}^{N \text { pixels }} \sum_{l}^{\text {Ncolors }} \mathrm{p}(\mathrm{i}, \mathrm{l})}
$$

5. Run Percentage (RP): This feature represents the percentage of runs in the opened window when normalized with respect to the radius $(\mathrm{R})$. The smaller the number of runs, the smaller the percentage, which means the window, has large homogeneous regions. Eq. (22) is used to generate RP.

$$
R P=\frac{100}{R} \sum_{i=1}^{N \text { pixels }} \sum_{l}^{N \text { colors }} \mathrm{p}(\mathrm{i}, \mathrm{l})
$$

B) Color based features

On the other hand, six color based features are extracted from each opened circular window. However; to represent the slide image in more intuitive and perceptually relevant manner, it will be converted to Hue Saturation Value (HSV) color model before color feature computation. Eqs. (23)(25) are used to convert Red Green Blue (RGB) color model to HSV color model [16].

$$
H= \begin{cases}\cos ^{-1}\left(\frac{0.5[(R-G)+(R-B)]}{\sqrt{(R-G)^{2}+(R-B)(G-B)}}\right) & \text { if } B \leq G \\ 2 \pi-\cos ^{-1}\left(\frac{0.5[(R-G)+(R-B)]}{\sqrt{(R-G)^{2}+(R-B)(G-B)}}\right) & \text { if } B>G\end{cases}
$$

$$
\begin{aligned}
& S=1-3 \times \operatorname{Min}(R, G, B) \\
& V=(R+G+B) /(3 \times 255)
\end{aligned}
$$

The following color features are then extracted from the HSV representation of each opened circular window:

1. Hue band mean: This feature represents the mean value of the dominant color in the opened window and can be calculated as follows:

$$
\text { Hmean }=\frac{1}{\text { Npixels }} \sum_{i=1}^{\text {Npixels }} H(i)
$$

2. Saturation band mean: This feature represents the mean value of the opened window brightness and can be calculated as follows:

$$
\text { Smean }=\frac{1}{\text { Npixels }} \sum_{i=1}^{N p i x e l s} S(i)
$$

3. Value band mean: This feature represents the mean value of the opened window intensities and can be calculated as follows:

$$
\text { Vmean }=\frac{1}{\text { Npixels }} \sum_{i=1}^{\text {Npixels }} V(i)
$$

4. Hue to saturation ratio: This feature represents the ratio of hue to saturation mean value of the opened window intensities and can be calculated as follows:

$$
H t S=\frac{\text { Hmean }}{\text { Smean }}
$$

5. Hue to value ratio: This feature represents the ratio of hue to value mean value of the opened window intensities and can be calculated as follows:

$$
H t V=\frac{\text { Hmean }}{\text { Vmean }}
$$

6. Saturation to value ratio: This feature represents the ratio of saturation to value mean value of the opened window intensities and can be calculated as follows:

$$
\mathrm{StV}=\frac{\text { Smean }}{\text { Vmean }}
$$

\subsection{Malaria infection detection}

Deep Belief Network (DBN) is used for final classification task. DBN is employed due to its strong ability because it performs unsupervised learning to determine the initial weights of the network and then starts the supervised learning. In this manner DBN can avoid the vanishing problem that may happen during leaning process and in turn leads to speed up the learning process.

\section{Experimental results and analysis}

\subsection{Database}

The images used in this work were constructed from the whole slide image that is provided by Pathology Education Instructional Resource (PEIR) repository at the University of Alabama at Birmingham, Department of Pathology [17]. One hundred images are built (50 positive images and 50 negative malaria infected images). To avoid over fitting during training process, $80 \%$ of samples within each class are used for system training and $20 \%$ for testing purpose.

\subsection{Results}

It is found that the best bright threshold is 200 and $\beta$ value is 0.9 . While the optimal $k$ value in corner detection is 0.05 and $T$ is 10000 . The best 
window size $(w s)$ in non-maximal suppression is 15 and Gabor filter bandwidth $\sigma$ is 2 . On the other hand; different experiments are conducted to study the effect of changing the value of $S c, R$, and $\theta$ parameters on the final accuracy of the proposed system. The classification accuracy can be computed as follows [18]:

$$
\text { Accuracy }=\frac{\text { correct }}{\text { Total }} \times 100
$$

Where correct represents the number of speech signals that correctly classified and Total is the total number of speech samples in the database.

Different experiments are conducted to find the optimal configuration of the proposed system parameters. The effectiveness of each local descriptor type is firstly tested after that the effectiveness of combining the two descriptor types is also given.

\section{A) Effectiveness of Gabor based features}

The purposed of this experiment is measuring the ability of Gabor based features in detection the infected slide image of blood cells. Tables 2-4 show the accuracy that is reached when attempting different numbers of corner points $(S c)$ and different Gabor orientation $(\theta)$ values for three different $R$ values.

Table 2. Accuracy achieved using Gabor based local features when $R=5$

\begin{tabular}{ccccc}
\multicolumn{5}{c}{ features when $R=5$} \\
\hline & $\boldsymbol{\theta}=\mathbf{4 5}$ & $\boldsymbol{\theta}=\mathbf{9 0}$ & $\begin{array}{l}\boldsymbol{\theta} \\
=\mathbf{1 3 5}\end{array}$ & $\boldsymbol{\theta}=\mathbf{1 8 0}$ \\
\hline$S_{c=5}$ & $93 \%$ & $96 \%$ & $91 \%$ & $93 \%$ \\
$S_{c=10}$ & $94 \%$ & $93 \%$ & $94 \%$ & $94 \%$ \\
$S_{c=15}$ & $94 \%$ & $93 \%$ & $92 \%$ & $94 \%$ \\
$S_{c=20}$ & $95 \%$ & $95 \%$ & $92 \%$ & $96 \%$ \\
\hline
\end{tabular}

Table 3. Accuracy achieved using Gabor based local features when $R=10$

\begin{tabular}{ccccc}
\hline & $\boldsymbol{\theta}=\mathbf{4 5}$ & $\boldsymbol{\theta}=\mathbf{9 0}$ & $\boldsymbol{\theta}=\mathbf{1 3 5}$ & $\begin{array}{l}\boldsymbol{\theta} \\
=\mathbf{1 8 0}\end{array}$ \\
\hline$S c=5$ & $90 \%$ & $93 \%$ & $84 \%$ & $92 \%$ \\
$S c=10$ & $92 \%$ & $96 \%$ & $91 \%$ & $94 \%$ \\
$S c=15$ & $92 \%$ & $96 \%$ & $92 \%$ & $96 \%$ \\
$S c=20$ & $94 \%$ & $94 \%$ & $91 \%$ & $95 \%$ \\
\hline
\end{tabular}

Table 4. Accuracy achieved using Gabor based local features when $R=15$

\begin{tabular}{ccccc}
\hline & $\boldsymbol{\theta}=\mathbf{4 5}$ & $\boldsymbol{\theta}=\mathbf{9 0}$ & $\boldsymbol{\theta}=\mathbf{1 3 5}$ & $\begin{array}{l}\boldsymbol{\theta} \\
=\mathbf{1 8 0}\end{array}$ \\
\hline$S_{c=5}$ & $91 \%$ & $94 \%$ & $87 \%$ & $92 \%$ \\
$S_{c=10}$ & $94 \%$ & $99 \%$ & $92 \%$ & $95 \%$ \\
$S_{c=15}$ & $93 \%$ & $96 \%$ & $95 \%$ & $93 \%$ \\
$S_{c=20}$ & $95 \%$ & $96 \%$ & $94 \%$ & $96 \%$ \\
\hline
\end{tabular}

Table 5. Accuracy achieved using color based local features

\begin{tabular}{cccc}
\hline & $R=5$ & $R=10$ & $R=15$ \\
\hline$S c=5$ & $67 \%$ & $54 \%$ & $53 \%$ \\
$S c=10$ & $88 \%$ & $72 \%$ & $73 \%$ \\
$S c=15$ & $73 \%$ & $71 \%$ & $61 \%$ \\
$S_{c=20}$ & $73 \%$ & $84 \%$ & $92 \%$ \\
\hline
\end{tabular}

Table 6. Accuracy achieved using combined local

\begin{tabular}{cccc}
\multicolumn{4}{c}{ features } \\
\hline & $R=5$ & $R=10$ & $R=15$ \\
\hline$S_{c=5}$ & $75 \%$ & $75 \%$ & $75 \%$ \\
$S_{c}=10$ & $100 \%$ & $100 \%$ & $100 \%$ \\
$S_{c}=15$ & $97 \%$ & $75 \%$ & $75 \%$ \\
$S_{c}=20$ & $95 \%$ & $95 \%$ & $94 \%$ \\
\hline
\end{tabular}

As shown in Tables 2-4, Gabor based features give accuracy ranged from $87 \%$ to $99 \%$ with respect to the different values of theta and number of selected corner points. However; the best accuracy (99\%) is achieved when $\theta=90$ and $S c=10$ and number of local features equals to $50(S c \times$ number of Gabor based features $=10 \times 5=50$ )

\section{B) Effectiveness of color based features}

Table 5 shows accuracy achieved using color based features with respect to different number of corner points and different radius of the opened window. The best accuracy achieved with color based features is $92 \%$ when $S c=20, R=5$ and feature vector of length equals to $20 \times 6=120$ features.

\section{C) Effectiveness of combined features}

Table 6 shows results that are obtained using feature vector consists of combined local descriptors (i.e., Gabor and color based features). As shown in the table, the desired accuracy which is $100 \%$ is achieved when $S c=10$ and $R=5$. This refers to the fact that combined types of descriptors can improve the accuracy by identifying more patterns about the micro regions of the blood slide image.

In addition, sensitivity and the specificity measures are used to further measure the performance of the proposed system. They can be calculated as in the following equations [12]:

$$
\begin{aligned}
& \text { Sensitivity }=\frac{T P}{T P+F N} \times 100 \\
& \text { Specificity }=\frac{T N}{T N+F P} \times 100
\end{aligned}
$$

where $T P$ refers to the number of true positive samples, $T N$ refers to the number of samples which are true negative, FP refers to the number of false positives samples, and $F N$ is the number of false 
Table 7. The calculated sensitivity and specificity values

\begin{tabular}{ccc}
\hline Feature Type & Sensitivity & Specificity \\
\hline Gabor based features & $100.00 \%$ & $98.00 \%$ \\
Color based features & $83.33 \%$ & $85.71 \%$ \\
Combined features & $100.00 \%$ & $100.00 \%$ \\
\hline
\end{tabular}

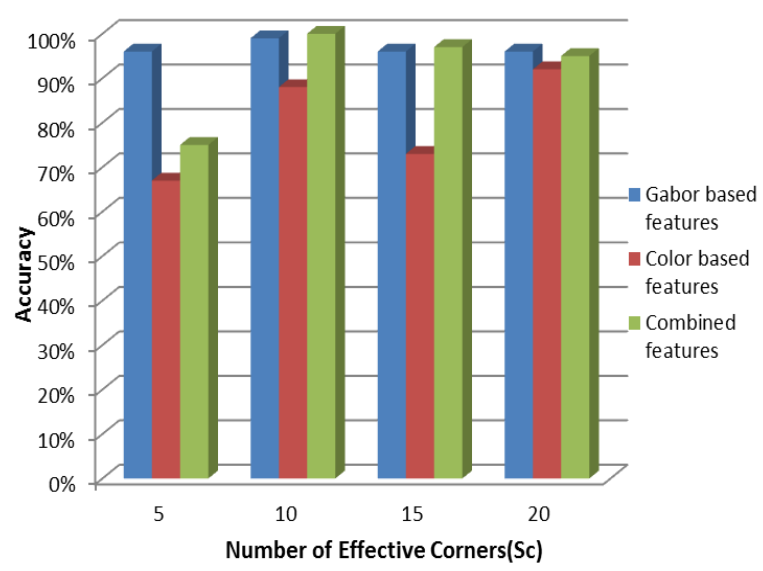

(a)

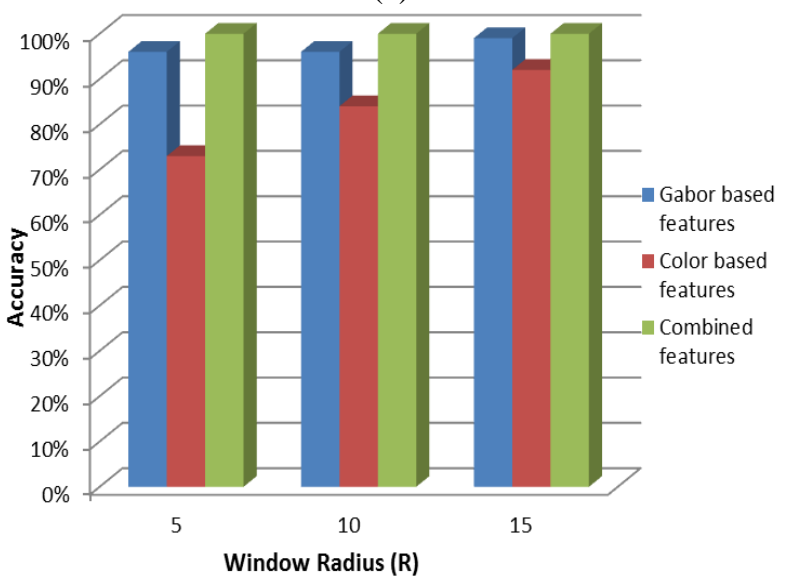

(b)

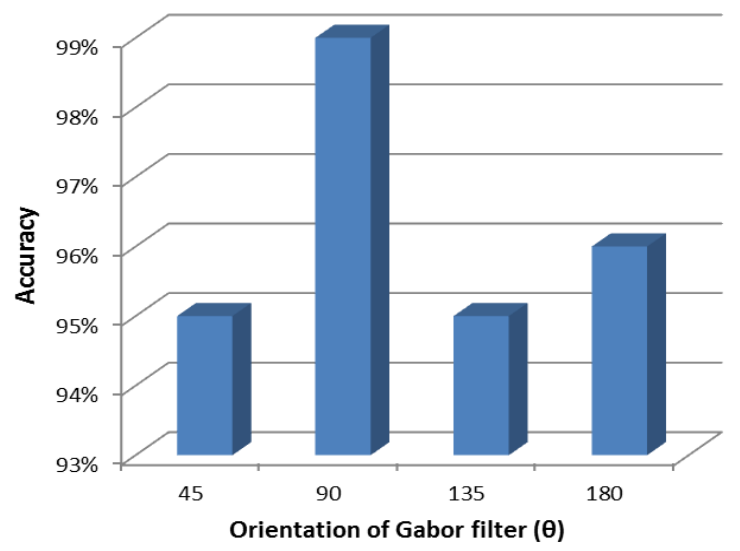

(c)

Figure. 4 The effect of system parameters on the resulted accuracy: (a) number of effective corners $(S c)$, (b) opened window radius $(R)$, and (c) orientation of Gabor filter $(\theta)$ negatives samples. The calculated specificity and sensitivity values for the cases that give the best accuracy within each local feature type are given in Table 7.

\subsection{Results analysis}

As shown in results section, when each of local descriptor type is used separately, the best accuracy of $99 \%$ is achieved using Gabor based features. On the other hand, the combination of the two descriptors type increases the accuracy to reach the desired accuracy which is $100 \%$. This refers to the fact that using color based features and the Gabor based features can lead to more effective feature vector.

On the other hand, as number of effective corners $(S c)$ and radius $(R)$ increase the accuracy also increases as shown in Fig. 4 (a) and (b). The main reason behinds that is the number of pixels in the opened windows will be increased which in effect enables the local features classifier to represent the texture involved within the opened window. However; when $R$ reached the value 5 and $S c=10$ in the combined descriptor, the accuracy reached $100 \%$ which refers to the fact of reaching optimal feature set for both of color based and Gabor based feature types.

The effect of Gabor filter orientation can be seen in Fig. 4 (c) and as it is clearly shown when $\theta=90$, the best accuracy is achieved.

\subsection{Comparison with other works}

Table 8 shows a comparison made between the proposed system and [9] which is based on the same database for evaluation purpose to ensure a fair comparison under the same conditions. The table also shows the used methods and achieved accuracy by each work. As it is clearly shown in the table, the results that are achieved by the proposed system outperform results of [9]. This reflects the effectiveness of the local based descriptors in capturing the micro details of the slide image and enhancing the infected blood smears detection.

Table 8. Comparison between the proposed system and other works

\begin{tabular}{|c|c|c|}
\hline Authors & Method & Achieved results \\
\hline $\begin{array}{l}\text { Pan et al. } \\
\text { (2018) } 9]\end{array}$ & CNN & Accuracy $=95.00 \%$ \\
\hline $\begin{array}{l}\text { The } \\
\text { Proposed } \\
\text { system }\end{array}$ & $\begin{array}{c}\text { PSO-based } \\
\text { automatic } \\
\text { segmentation, } \\
\text { Color and Gabor } \\
\text { based local } \\
\text { descriptors and } \\
\text { DBN }\end{array}$ & $\begin{array}{l}\text { Accuracy }=100 \% \\
\text { sensitivity }=100 \% \\
\text { specificity }=100 \%\end{array}$ \\
\hline
\end{tabular}




\section{Conclusion}

An intelligent system has been presented in this paper to identify the infected malaria blood cells based on local descriptors of interested corners' points. The PSO-based segmentation method helps in determining the optimal threshold value in adaptive manner based on the transparency between the blood cells and the background. The local micro patterns in the texture of blood smear image are obtained by using local descriptors which in effect leads to more effective features. Deep belief network also gives the proposed system the power in understanding the hidden patterns within the generated descriptors. Experimental results showed that an accuracy of $100 \%$ was achieved using feature vector consist of a combination from Gabor and color based local features. As future work, other types of local descriptors (such as fractal features) can be used to measure their effectiveness in identifying the infected blood smear image.

\section{Conflicts of Interest}

The authors declare no conflict of interest.

\section{Author Contributions}

Conceptualization, Yossra H. Ali and Suhaila N. Mohammed; methodology, Yossra H. Ali; software, Suhaila N. Mohammed; validation, Yossra H. Ali; formal analysis, Suhaila N. Mohammed; investigation, Yossra H. Ali; resources, Suhaila N. Mohammed; data curation, Suhaila N. Mohammed; writing-original draft preparation, Suhaila N. Mohammed; writing_review and editing, Yossra $\mathrm{H}$. Ali; visualization, Yossra H. Ali; supervision, Yossra H. Ali; project administration, Yossra H. Ali; funding acquisition, Yossra H. Ali.

\section{References}

[1] M. Poostchi, K. Silamut, R. Maude, S. Jaeger, and G. Thoma, "Image Analysis and Machine Learning for Detecting Malaria", Translational Research, Vol. 194, pp. 36-55, 2018.

[2] World Malaria Report 2019, https://www.who.int/publications/i/item/worldmalaria-report-2019

[3] F. Tek, A. Dempster, and I. Kale, "Computer Vision for Microscopy Diagnosis of Malaria", Malaria Journal, Vol. 8, No. 153, pp. 1-14, 2009.

[4] A. Loddo, C. Ruberto, and M. Kocher, "Recent Advances of Malaria Parasites Detection Systems Based on Mathematical Morphology", Sensors, Vol. 18, No. 513, pp. 1-21, 2018.
[5] N. Lepore, J. Brieva, E. Romero, D. Racoceanu, and L. Joskowicz, Processing and Analysis of Biomedical Information, Springer, 2019.

[6] M. Mushabe, R. Dendere and T. Douglas, "Automated Detection of Malaria in GiemsaStained Thin Blood Smears", In: Proc. of 35th Annual International Conf. of the IEEE EMBS, Osaka, Japan, 2013.

[7] S. Savkare, and S. Narote, "Automated System for Malaria Parasite Identification", In: Proc. of 2015 International Conf. on Communication, Information \& Computing Technology (ICCICT), Mumbai, India, 2015.

[8] A. Bashir, Z. A. Mustafa, I. Abdelhameid, and R. Ibrahem, "Detection of Malaria Parasites Using Digital Image Processing", In: Proc. of 2017 International Conf. on Communication, Control, Computing and Electronics Engineering (ICCCCEE) Khartoum, Sudan, 2017.

[9] W. Pan, Y. Dong, and D. Wu, "Classification of Malaria-Infected Cells Using Deep Convolutional Neural Networks", In: Proc. of H. Farhadi (eds) Machine Learning, Intechopen, 2018.

[10] Q. Quan, J. Wang, and L. Liu, "An Effective Convolutional Neural Network for Classifying Red Blood Cells in Malaria Diseases", Interdiscip Sci Comput Life Sci, Vol. 12, pp. 217-225, 2020.

[11] S. Mohammed, A. Jabir, and Z. Abbas, "SpinImage Descriptors for Text-Independent Speaker Recognition", In: Proc. of Saeed F., Mohammed F., Gazem N. (eds) Emerging Trends in Intelligent Computing and Informatics. IRICT 2019. Advances in Intelligent Systems and Computing, Vol. 1073, Springer, Cham, 2019.

[12] S. Mohammed, F. Alkinani, and Y. Hassan, "Automatic Computer Aided Diagnostic for COVID-19 Based on Chest X-Ray Image and Particle Swarm Intelligence", International Journal of Intelligent Engineering and Systems, Vol. 13, No. 5, pp. 63-73, 2020.

[13] Z. Li, C. Liu, G. Liu, Y. Cheng, X. Yang, and C. Zhao, "A Novel Statistical Image Thresholding Method", International Journal of Electronic Communication (AEU), Vol. 64, No. 12, pp. 1137-1147, 2010.

[14] C. Harris and M. Stephens, "A Combined Corner and Edge Detector", In: Proc. of the Alvey Vision Conf., Vol. 23, pp. 1-6, 1988.

[15] A. Younesi and M. Amirani, "Gabor Filter and Texture based Features for Palmprint Recognition", In: Proc. of International Conf. 
on Computational Science, ICCS, Zurich, Switzerland, pp. 2488-2495, 217.

[16] S. Mohammed and H. Rada, "English Numbers Recognition Based On Sign Language Using Line-Slope Features and PSO-DBN Optimization Method", Journal of Engineering Science and Technology, Vol. 15, No. 3, pp. 1855-1867, 2020.

[17] PEIR-VM Website, http://peirvm.path.uab.edu/wsi.php?slide=IPLab11Malari a

[18] A. Hassan and S. Mohammed, "A Novel Facial Emotion Recognition Scheme Based on Graph Mining", Defence Technology, Vol. 16, No. 5, pp. 1062-1072, 2020. 